ÉGYPTE monde arabe

\section{Égypte/Monde arabe}

$12 \mid 2015$

Evolution des systèmes médiatiques après les révoltes arabes

\title{
La scolarisation des jeunes réfugiés palestiniens par l'Unesco/UNRWA de 1949 aux années 1980
}

\section{Chloé Maurel}

\section{OpenEdition}

\section{Journals}

Édition électronique

URL : https://journals.openedition.org/ema/3476

DOI : $10.4000 /$ ema.3476

ISSN : 2090-7273

Éditeur

CEDEJ - Centre d'études et de documentation économiques juridiques et sociales

Édition imprimée

Date de publication : 25 mars 2015

Pagination : 255-277

ISBN : 9782905838858

ISSN : 1110-5097

Référence électronique

Chloé Maurel, « La scolarisation des jeunes réfugiés palestiniens par l'Unesco/UNRWA de 1949 aux années 1980 », Égypte/Monde arabe [En ligne], 12 | 2015, mis en ligne le 25 mars 2015, consulté le 07 juillet 2022. URL : http://journals.openedition.org/ema/3476 ; DOI : https://doi.org/10.4000/ema.3476 


\section{Chloé Maurel}

\section{RÉSUMÉ / ABSTRACT}

Dès 1949, l'Unesco et l'Unrwa se sont engagées dans une action en faveur de l'éducation des jeunes réfugiés palestiniens, dans la Bande de Gaza, en Cisjordanie, en Jordanie, au Liban et en Syrie. Malgré les difficultés immenses dues à la situation de tension et même par moments de guerre entre les deux communautés ennemies, le programme éducatif Unrwa/Unesco a, de sa création en 1949 jusqu'à nos jours, réussi à mettre sur pied un véritable système scolaire pour les jeunes Palestiniens. Les conflits entre les deux camps se sont matérialisés notamment dans le contenu des manuels scolaires. Les moments de conflits violents (1956, 1967, 1974 et à partir de 1982 en particulier) ont perturbé gravement le fonctionnement de ce système mais, malgré les nombreuses difficultés, notamment financières, ce programme a toujours continué à œuvrer à la scolarisation des jeunes Palestiniens.

\section{MOTS CLÉS :}

Palestine, Israël, ONU, Unesco, éducation, réfugiés, histoire, $X X^{e}$ siècle, monde arabe.

Beginning in 1949, Unesco and UNRWA launched an educational program for young Palestinian refugees in the Middle East. In spite of the huge difficulties due to the Israeli-Palestinian conflict, this program succeeded in setting up a real educational system for young Palestinians.

\section{KEYWORDS:}

Palestine, Israel, United Nations, education, refugees, history, $X X^{\text {th }}$ century, arab world.

CHLOÉ MAUREL est normalienne, agrégée d'histoire, docteure en histoire contemporaine, auteure d'une thèse de doctorat sur l'histoire de I'Unesco (université Paris 1), chercheuse associée à I'IHMC (CNRS/ENS). A publié notamment Histoire de I'Unesco. Les trente premières années (L'Harmattan, 2010), Histoire des relations internationales depuis 1945 (Ellipses, 2010), Manuel d'histoire globale (Armand Colin, 2014).

Email : Chmaurel@yahoo.fr 


\section{Chloé Maurel}

\section{LA SCOLARISATION DES JEUNES RÉFUGIÉS PALESTINIENS PAR L'UNESCO/ UNRWA DE 1949 AUX ANNÉES 1980}

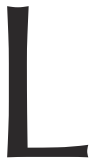

e 31 octobre 2011, I'Unesco a admis la Palestine comme Etat membre, ouvrant la voie à une reconnaissance de cet Etat par la communauté internationale. En réalité, l'intérêt de l'Unesco pour la Palestine ne date pas d'hier: en 1974 déjà, cette institution internationale avait admis l'Organisation de libération de la Palestine (OLP) comme observateur permanent en son sein. Mais l'action de l'Unesco en faveur des Palestiniens remonte en fait encore plus loin : en 1949, quelques années à peine après sa création, I'Unesco s'est engagée dans une action en faveur de l'éducation des enfants réfugiés palestiniens, dans la Bande de Gaza, en Cisjordanie, en Jordanie, au Liban et en Syrie. Il s'agissait de permettre à ces enfants d'être scolarisés malgré leur condition de réfugiés. L'organisation a mené cette action conjointement avec I'UNRWA, Office de secours et de travaux des Nations Unies pour les réfugiés de Palestine dans le Proche-Orient (United Nations Relief and Works Agency for Palestine Refugees in the Near East), créé à cet effet.

Créé d'abord de manière temporaire suite à la première guerre israélo-arabe de 1948 par la résolution 302 (IV) de l'Assemblée générale des Nations Unies du 8 décembre 1949, I'UNRWA s'est perpétué jusqu'à nos jours. Ce programme s'occupe des réfugiés palestiniens en matière de santé, d'aide humanitaire, de services sociaux et surtout d'éducation. Pour le domaine éducatif (qui concerne plus de $70 \%$ du personnel de ce programme), I'UNRWA travaille en coopération avec l'Unesco.

Selon la définition de l'UNRWA, un « réfugié de Palestine » est une personne dont le lieu de résidence habituelle était la Palestine entre juin 1946 et mai 1948 et qui a perdu à la fois son domicile et ses moyens de subsistance en raison du conflit israélo-arabe de 1948. La 
définition de réfugié de I'UNRWA couvre également les descendants des Palestiniens qui sont devenus des réfugiés en 1948. Ainsi, le nombre de réfugiés palestiniens enregistrés est passé de 700000 en 1950 à plus de 4,8 millions en 2005.

Comment le programme UNRWA-Unesco s'est-il créé et développé ? Quelles ont été les difficultés ? Quel est le bilan de ce programme? Il est intéressant de suivre le développement et les péripéties de ce programme, de sa création en 1948 jusqu'au milieu des années 1980, époque couverte par les archives de I'Unesco ouvertes à la consultation.

\section{LA MISE EN PLACE DU PROGRAMME}

Les premiers efforts de secours éducatif aux enfants palestiniens réfugiés sont entrepris dès I'hiver 1948-49 par différents organismes. L'UNRWA est établi en décembre 1949, sous l'autorité directe de l'assemblée générale de I'ONU. Son rôle est alors de distribuer des secours dans le domaine de la santé, de l'assistance sociale, de l'enseignement et de la formation professionnelle, aux réfugiés palestiniens établis en Jordanie, au Liban, en Égypte, en Syrie et se trouvant dans le besoin. L'Unesco s'associe à l'UNRWA pour I'action éducative auprès des enfants réfugiés. Aux termes de l'accord UNRWAUnesco, I'Unesco assume la responsabilité technique du programme d'éducation pour les réfugiés palestiniens, et l'UNRWA la responsabilité administrative.

Le système d'écoles primaires Unesco-UNRWA, mis en place dans un contexte d'urgence et d'improvisation, touche initialement une très faible proportion des enfants. Dans les premières années, les cours sont donnés dans des tentes et des bâtiments abandonnés. En quelques années, les effectifs augmentent rapidement. De nombreuses écoles sont construites dans les années 1950¹.

En 1951, le programme UNRWA/Unesco compte un peu plus de 42000 élèves dans 104 écoles avec 670 enseignants². Dès 1953, I'Unesco observe les effets positifs de son action, constatant que pour ces enfants, l'école est « l'élément le plus agréable du cadre de la vie quotidienne », et qu'elle leur " permet d'échapper à l'ennui généralisé

1. Knud Mortensen, K.D. Wagner, op.cit., p. 27-28, 30, 51, 65, 67 ; archives de I'Unesco, ED/131, 31 août 1953, p. 4 ; 37 : 362.92 (5-011) «-66 », Illa : lettre de Walter à van Vliet, $1^{\text {er }}$ mars 1958.

2. Archives de I'Unesco, dossier 37:362.92 (5-011) UNRWA/A 045 UNDP, part I : Doc Institute of Education, Beyrouth. 
qui caractérise la vie du réfugié $»^{3}$. Entre les deux visites qu'il accomplit sur place en 1952 et 1955, Lionel Elvin, fonctionnaire du département de l'éducation de I'Unesco, est impressionné du remplacement, en l'espace de quelques années, de la plupart des tentes par des bâtiments en dur munis de tous les équipements nécessaires; il estime que $d^{\prime}$ « immenses progrès » ont été faits ${ }^{4}$.

Le programme Unesco-UNRWA suscite l'enthousiasme de I'opinion des États arabes ${ }^{5}$, des articles favorables dans la presse française, britannique, américaine, et la satisfaction de I'Unesco ${ }^{6}$. Malgré d'importants problèmes financiers, politiques, et administratifs, qui persistent tout au long des années, ce programme s'efforce d'étendre peu à peu son ampleur. Ainsi, à partir de 1952, I'UNRWA s'efforce d'introduire une éducation secondaire en Jordanie, avec difficulté. L'éducation secondaire reste beaucoup moins développée que l'éducation élémentaire. La mise en place d'un système d'éducation secondaire et professionnelle se heurte aussi au problème de l'absence de débouchés professionnels. Malgré les efforts de I'Unesco notamment par la mise en place de nombreuses écoles professionnelles, les jeunes réfugiés ont du mal à trouver un emploi ${ }^{7}$. La formation des enseignants se heurte elle aussi à d'importantes difficultés ${ }^{8}$, malgré la création à Beyrouth d'un «institut pédagogique de formation des maîtres en exercice », et la mise en place d'écoles normales.

3. Archives de I'Unesco, ED/131, 31 août 1953, p. 1 ; archives de I'ONU, RAG 2/76 box 7: instructions d'Adiseshiah aux experts d'assistance technique sur le terrain, 1er juin 1953, p. 9-10.

4. Archives de I'Unesco, dossier de correspondance $37: 362.92$ (5-011) «-66 », Illa : rapport confidentiel de L. Elvin, 3 oct. 1955, p. 1.

5. Archives diplomatiques américaines, Decimal file, RG59, entry CDF 1945-49 (NND 760050, from 501.PA/3-147 to 501.PA/4-1647) : box 2254 : Le Journal d'Egypte, 25 nov. 1948, éditorial : " L'Unesco s'ouvre aux hommes et aux choses du Moyen Orient»; archives de I'Unesco, ED/131, 31 août 1953, p. 4.

6. Archives de l'Unesco, revue de presse du 8 mai 1951 : Le Monde, 21 avril 1951 ; revue de presse du 19 mai 1953 : Revue de Paris, mai 1953; revue de presse du 10 août 1951: The Scotsman, Edimbourg, 31 juill. 1951 : «Impressive work of Unesco. Education of Arab refugee Children- 40 schools set up », par Philip Toynbee.; New York Times, 15 mars 1949, p. 11 : "Education is Urged for Arab Refugees »; dossier de correspondance $37: 362$ .92 (5-011) «-66 », Illa : rapport confidentiel de L. Elvin, 3 oct. 1955, p. 2.

7. Archives de I'Unesco, dossier de correspondance $37: 362.92$ (5-011) «-66 », IV : mémo confid. du DG p.i. à McCune, 24 juill. 1961.

8. Archives de I'Unesco, dossier de correspondance $37: 362.92$ (5-011) "-66 », IV : rapport confidentiel de W.H. Loper au DG, 2 janv. 1959, p. 6-10 ; mémo de Maheu à Aleksander, 27 juill. 1959; rapport confid. de W.A. Mustakim au DG, 12 oct. 1960 ; lettre de J. Guiton au DG p.i., 13 juill. 1961. 


\section{L'ORGANISATION DES EXAMENS}

En 1969, I'Unesco réussit à conclure avec les gouvernements concernés une série $d^{\prime}$ accords qui aboutissent à l'organisation dans la bande de Gaza, à partir de juillet 1969, d'examens de fin d'études secondaires auxquels plus de 7000 candidats se présentent. L'Unesco se charge de leur déroulement, du transport des sujets, des copies et des diplômes entre l'Egypte (où les sujets sont élaborés, où les copies sont corrigées, et où les diplômes sont émis) et la bande de Gaza, où les examens ont lieu. Ce système se poursuit dans les années suivantes. En outre, sur l'appel de René Maheu, directeur général de I'Unesco, les gouvernements égyptien, libanais et syrien décident à partir de 1971 d'accorder des bourses d'études supérieures à des étudiants réfugiés de Gaza. Ce système d'examens mis en place par I'Unesco est un succès et entraîne des échos admiratifs dans la presse mondiale ${ }^{9}$. Au total, le programme Unesco-UNRWA a dans les années 1950 et 1960 permis un accroissement colossal de la scolarisation des jeunes réfugiés.

En juillet 1971, 7000 candidats se présentent aux examens de fin d'étude secondaire, 3418 sont reçus. Le gouvernement égyptien offre 1000 bourses pour des étudiants de la bande de Gaza pour l'année universitaire 1971-72. Le Comité international de la Croix-Rouge (CICR) organise le transport de ces étudiants dans cinq convois qui traversent le canal de Suez en août-septembre 1971.

L'Unesco-UNRWA continue, tout au long des années 1970, à superviser les examens, et à donner des bourses à des étudiants palestiniens . Le nombre des élèves qui se présentent à cet examen varie de 7000 à 9000 par an. Environ 1000 élèves parmi ceux qui réussisent chaque année cet examen poursuivent des études supérieures dans les universités de la RAU.

\section{DES TENSIONS POLITIQUES}

Dans une lettre confidentielle de 1955, Lionel Elvin reconnaît que «bien que le travail éducatif de l'UNRWA soit strictement nonpolitique (...), il s'inscrit inévitablement dans le contexte malheureux de la situation politique », et que ce programme pâtit de son manque

9. Archives de I'Unesco, revue de presse du 9 fév. 1970 : The Guardian, Londres, 2 janv. 1970 ; RP, 31 oct. 1969 : Le Monde, 4 oct. 1969 : "Grâce à I'Unesco - Plus de 7000 étudiants de la région de Gaza ont p. passer leurs examens »; New York Times, 3 oct. 1969. 
de reconnaissance officielle par les États arabes, puisque «pour les États arabes, reconnaître le projet signifie reconnaître la permanence des réfugiés à l'intérieur de leurs frontières et amène logiquement à la reconnaissance des 'territoires palestiniens occupés' en Israël ${ }^{10}$. Les tensions accrues entre Israël et les pays arabes en 1956-57 entravent gravement le déroulement du programme. Israël impose un contrôle drastique sur les manuels scolaires dans les camps des territoires qu'elle occupe $^{11}$. En 1958, le contexte politique tendu perturbe le programme, qui se déroule dans une atmosphère de danger, au milieu des menaces et de l'enlèvement de professeurs ${ }^{12}$.

Dès le début et tout au long des années, les États-Unis ne sont pas favorables au programme Unesco-UNRWA, jugé d'esprit hostile à Israël ${ }^{13}$. Durant la guerre israélo-palestinienne de 1967 (guerre des Six Jours), ce programme connaît des problèmes liés à I'occupation par Israël de la rive droite du Jourdain et de la bande de Gaza où se trouvaient $40 \%$ des 1500000 réfugiés recensés par l'UNRWA.

En septembre 1970, John S. Rennie, assistant commissaire général du programme Unesco-UNRWA, écrit au directeur général (DG) de I'Unesco, René Maheu, et lui confie les " graves difficultés » vécues au centre de formation de Siblin au Liban pendant I'année scolaire

10. Archives de I'Unesco, dossier de correspondance $37: 362.92$ (5-011) «-66», III a : confid. report of visit to Beirut, UNRWA-Unesco schools, 3 oct. 1955, 6 p., par Lionel Elvin, p. 2.

11. Archives de l'Unesco, dossier de correspondance $37: 362.92$ (5-011) «-66»: III a : mémorandum de conversation, par Van Vliet, avec Lewin, délégué permament adjoint d'Israël à I'Unesco, 5 sept. 1957 ; lettre de Van Vliet à B. Aleksander, 14 mars 1958.

12. 37 : $362.92(5-011)$ «-66», IV : rapport confid. de Van Diffelen à Van Vliet, 5 nov. 1958.

13. Rapports du Congrès américain, vol. 112, 89e congrès, 2e session, 10 janv. -22 oct. 1965 : 24 août 66, House : "UNRWA misuse of US funds ", p. 20423-20424. Intervention de Mr. Friedel, du Maryland. « various substantial abuses in the UNRWA operations »; " hatred directed against the West and particularly against the US and the State of Israël »; Baltimore Jewish Times, 29 juill. 1966 : " How Stupid Can One Get? », par Eliezer Whartman ; éditorial, "We Accuse UNRWA ", in Bulletin of the Society for the prevention of World War III, janvier-mars 1971. Articles cités dans: Congrès, vol. 116, 91e congrès, 2e session, 19 janv. 1970-2 janv. 1971 : 27 février 1970, Sénat, p. 5264-5265: "Should UN funds finance the training of Arab terrorists?", Mr Dodd. " training grounds for Arab guerillas ». Congrès, Vol. 115, 91e congrès, 1e session, 3 janv.-23 déc. 1969 : UNRWA, 35210. Congrès, Vol. 116, 91e congrès, 2e session, 19 janv. 1970-2 janv. 1971 : UNRWA, 39153, 39151, 39154, 39157 ; "We accuse UNRWA », 5264, 12298. Congrès, Vol 117, 92e congrès, 1e session, 21 janv.-17 déc. 1971 : UNRWA, p. 15117. 
1969-1970. Les difficultés concernent principalement le maintien de la discipline et sont liées à l'état troublé de la communauté des réfugiés palestiniens au Liban. Les étudiants ont lancé des grèves à de nombreuses occasions. John S. Rennie juge l'état de marche du centre " très insatisfaisant ». Il déplore une forte "indiscipline » qui aurait dégénéré en "vandalisme " à la fin de l'année scolaire et signale qu'on aurait trouvé des armes à feu et des munitions dans le centre ${ }^{14}$. Cet exemple témoigne des grandes difficultés vécues au quotidien dans les écoles et les centres de formation Unesco-UNRWA.

\section{LE PROBLÈME DES MANUELS}

La question des manuels pose des difficultés dans les zones occupées par Israël ; les autorités israéliennes bannissent l'usage de la plupart des manuels qui étaient alors en vigueur dans les écoles de réfugiés de la bande de Gaza, au motif que ces manuels inciteraient à la haine. L'Unesco décide alors, en 1968, de faire réviser ces manuels par une commission de personnalités extérieures ${ }^{15}$. Livres d'arabe, de religion, de sciences, etc. sont contrôlés et expurgés des passages jugés anti-sionistes ${ }^{16}$. Beaucoup de manuels s'avèrent teintés $d^{\prime} u n e$ forte propagande anti-sioniste ${ }^{17}$. Ce problème des manuels, très politisé, persiste dans les années qui suivent. En 1970, René Maheu accuse Israël d'empêcher l'entrée dans les territoires occupés arabes de manuels destinés aux écoliers palestiniens. Israël obtiendra par la suite que soient retirés des bibliothèques scolaires palestiniennes des ouvrages jugés haineux à l'égard de l'État israélien. En 1975 encore, beaucoup d'élèves de la bande de Gaza sont dépourvus des manuels scolaires car les autorités d'occupation israéliennes interdisent l'entrée de ces livres, qui sont imprimés en Egypte ${ }^{18}$. Alors, devant l'impasse créée par le refus des Israéliens d'accepter les manuels, I'Unesco met en place une nouvelle commission composée d'experts extérieurs ; ils étudient les manuels, puis le DG autorise les manuels, plusieurs

14. Archives de I'Unesco, dossier de correspondance 37 :362.92 (5-011)

(074) part II : lettre de John S. Rennie, A/Commissioner general, à Maheu, 23 septembre 1970.

15. Archives de I'Unesco, DG/68/8, 10 juill. 1968, p. 5-6.

16. Archives de I'Unesco, dossier 37:362.92 (5-011) A 312 (569.433+62) UNRWA part II.

17. Archives de I'Unesco, dossier 37:362.92 (5-011) A 312 (569.433+62) UNRWA part II.

18. Archives de l'Unesco, dossier 37:362.92 (5-011) UNRWA « 67 -», part VIII : note du 17 juill 75 : Mise en œuvre de la résolution concernant l'éducation et la culture dans les territoires arabes occupés. 
sont révisés ${ }^{19}$. Au fil des années, se poursuit une correspondance entre I'Unesco et les autorités israéliennes sur les autorisations de manuels ${ }^{20}$.

\section{DE DURES CONDITIONS DE VIE ET D'ÉTUDE POUR LES ÉLÈVES ET LES ENSEIGNANTS}

En 1970, un agent de I'ONU, dans une "Note sur les aspects psychologiques de l'occupation » dans les écoles de I'UNRWA-Unesco dans les territoires occupés de la Cisjordanie et de Gaza, observe que les autorités israéliennes maintiennent l'ordre et répriment la résistance par toutes sortes de mesures sévères, "souvent juridiquement et moralement indéfendables: vexations policières, arrestations, déportations, condamnations à de lourdes peines de prison » etc. ${ }^{21}$ II observe dans les écoles et les centres de I'UNRWA/Unesco, des arrestations de maîtres ou d'étudiants, des déportations de maîtres, des couvre-feu fréquents qui entravent la fréquentation normale des écoles, la fermeture temporaire de certaines écoles, des coups et blessures infligés à un grand nombre enfants (94 jeunes filles blessées en février 1969), des restrictions nombreuses à la liberté de mouvement et à la liberté de l'enseignement.

Il analyse l'état d'esprit des habitants des territoires occupés : ces derniers, écrit-il, supportent généralement « avec courage » les épreuves de l'occupation, mais qu'ils n'en sont pas moins "constamment tendus, nerveux et inquiets » et ils ont développé une méfiance permanente. Il observe que le danger et le malheur vécus en commun ont renforcé les sentiments de solidarité, et que depuis la guerre des Six Jours ils manifestent un véritable "vouloir-vivre collectif». Ainsi, malgré, ou peut-être à cause des difficultés dues aux fortes tensions, les Palestiniens montrent, selon ce rapport, une énergie remarquable et un comportement solidaire.

Concernant les fonctionnaires palestiniens de I'UNRWA, il observe qu'ils « connaissent un problème particulier » : en tant que membres d'une agence internationale, ils ont à s'abstenir de toute activité de nature politique; mais « en tant que Palestiniens, conscients d'être les victimes d'une grave injustice, ils ne peuvent pas ne pas se sentir profondément concernés par les événements qui les entourent. Ils ont

19. Archives de I'Unesco, dossier 37:362.92 (5-011) A 06, part IV : texte envoyé par Mustakim au DG, 25 septembre 1975 : intitulé « L'Unesco et le programme d'aide en matière d'éducation aux réfugiés palestiniens ».

20. Archives de I'Unesco, dossier 37:362.92 (5-011) (074), part IX.

21. Archives de I'Unesco, dossier 37:362.92 (5-011) UNRWA « 67 -», part VI : Note de Paul Esseiva, ONU, 11 juillet 1970. 
donc à refréner sans cesse leurs sentiments nationaux, et à essayer de trouver la juste limite entre des obligations contradictoires»; "leur position est hautement inconfortable ».

Il observe aussi que dans les écoles, l'atmosphère est souvent " nerveuse et tendue », et que les manifestations et grèves scolaires placent les maîtres dans une situation difficile. "Comme patriotes palestiniens, ils approuvent peut-être ce qu'ils devraient désapprouver soit comme éducateurs, soit comme membres d'une agence internationale $»^{22}$. La situation des enseignants est donc particulièrement inconfortable et périlleuse.

Cependant, malgré ces problèmes, plusieurs éléments apparaissent positifs. Pendant l'année scolaire 1972-73, 260000 enfants sont inscrits dans les écoles UNRWA-Unesco, presque la moitié sont des filles. II y a environ 7000 professeurs, dont presque la moitié sont des femmes ${ }^{23}$. La forte participation d'une population féminine est un succès à mettre au crédit de ce programme. De plus, en 1973, un spécialiste de l'OMS, qui fait une étude auprès des enfants du programme Unesco-UNRWA, observe qu'un nombre inhabituellement faible d'entre eux ont des problèmes mentaux ${ }^{24}$, ce qui est aussi un élément positif.

\section{VERS LA CRÉATION D'UNIVERSITÉS POUR LES PALESTINIENS}

En 1964 est créé un institut éducatif UNRWA/Unesco à Beyrouth. II sert à former des enseignants. Par ailleurs, à l'époque de l'administration jordanienne, jusqu'en juin 1967, il n'y avait aucune université dans la région de Judée-Samarie. La seule université de Jordanie se trouvait à Amman, c'était un établissement public. Depuis 1967, la JudéeSamarie relève de I'administration israélienne, plusieurs universités privées sont créées. En réponse à des demandes des représentants de la population, les autorités israéliennes autorisent la création de quatre universités : I'université de Beir-Zeit (fondation privée, située près de Ramallah, affiliée à I'université américaine de Beyrouth), I'université nationale d'Al-Najah, I'université de Bethléem, le centre d'études islamiques (college al Shahryah).

22. Archives de I'Unesco, dossier 37:362.92 (5-011) UNRWA « 67 -», part

VI : Note de Paul Esseiva, ONU, 11 juillet 1970.

23. Archives de I'Unesco, dossier $37: 362.92$ (5-011) «67--» : Lettre de JS Rennie à W Mustakim, 27 mars 1973.

24. Archives de l'Unesco, dossier $37: 362.92$ (5-011) « 67- -» : Lettre de JS Rennie à A Varchaver, 13 avril 1973. 
En 1970, une initiative constructive est lancée : celle de mettre en place une nouvelle université arabe pour les réfugiés palestiniens. Des experts arabes et israéliens travaillent de concert à ce projet. En 1973 l'établissement d'une université arabe de Palestine en Cisjordanie est un pas en avant important. L'université de Bethléem ouvre en septembre 1973. A son ouverture, 80 étudiants y sont inscrits. A la demande du Vatican, la direction de l'université est assurée par les frères américains des écoles chrétiennes. La mission pontificale en Terre sainte et la hiérarchie épiscopale allemande en assurent l'essentiel du financement. En fait, les racines de cette université remontent à 1893 quand les Frères des Écoles chrétiennes ont fondé des écoles dans la région.

En 1977, à la demande du fonds arabe pour le développement économique et social et de l'OLP, le secrétariat de l'Unesco prépare une étude de pré-faisabilité sur la création d'une université ouverte palestinienne. Puis une seconde étude de faisabilité est réalisée en 1980. En novembre 1980, I'assemblée générale de I'ONU adopte une résolution demandant l'étude, notamment par I'Unesco, de la création à Jérusalem, sous les auspices de l'ONU, d'une université comporté des facultés des lettres et des sciences pour répondre aux besoins des réfugiés de Palestine dans la région. En 1981, dans cette étude de faisabilité, I'Unesco estime que 40000 jeunes arabes palestiniens achèvent avec succès leurs études secondaires tous les ans, et que sur ce chiffre seuls 10000 entrent à l'université, et que les 30000 autres ne peuvent poursuivre leurs études au niveau universitaire essentiellement du fait du manque d'établissements universitaires. L'Unesco observe que cette situation est amenée à s'aggraver pour des raisons démographiques et du fait du nombre croissant de filles faisant des études ${ }^{25}$. En novembre 1982 un rapport du secrétaire général de I'ONU conclut donc à l'urgence d'établir une université à Jérusalem ${ }^{26}$. Cela aboutira finalement à la création de I'Université ouverte d'AlQuds, créée en 1991 à Amman. Quant à I'université de Beir Zeit, en 1985 les force israéliennes s'en emparent, prenant alors en main les admissions et l'embauche de personnel. L'université fermera ses portes pendant la première Intifada.

Ainsi, le succès du programme de scolarisation Unesco/UNRWA n'a pas tardé à susciter le besoin de créer des universités ouvertes aux

25. Archives de l'Unesco, dossier 37:362.92 (5-011) UNRWA/PLO part III : doc. A/36/593, 30 oct 1981 : assemblée générale de l'ONU.

26. Archives de l'Unesco, dossier 37:362.92 (5-011) UNRWA/PLO part IV : doc. A/37/599, 5 nov 1982 : question of the establishment of a university at Jerusalem; report of the secretary general. 
Palestiniens, ce qui est fait avec le soutien des institutions onusiennes. Mais certaines de ces universités cristallisent l'hostilité entre Israéliens et Palestiniens.

\section{DE GRAVES DIFFICULTÉS FINANCIÈRES EN 1971-72}

En 1970-71, le programme UNRWA/Unesco connaît de graves difficultés financières. Au point que le DG René Maheu lance le 1er janvier 1971 un appel solennel à des dons en faveur de ce programme. Des Etats répondent en faisant des dons, principalement des Etats européens et des Etats arabes. Les Etats-Unis, rappelant qu'ils contribuent pour près de $70 \%$ des ressources reçues par UNRWA des gouvernements, ne versent pas d'argent suite à cet appel ${ }^{27}$. L'Unesco envoie aussi directement des lettres à des entreprises et des fondations privées pour leur demander des dons pour ce programme. Grâce à cet appel, I'Unesco parvient, en cinq mois, à enregistrer 1200000 dollars de contributions supplémentaires au programme. Mais cette somme est loin de combler le déficit du programme ${ }^{28}$. Cette annéelà, le programme comporte 500 écoles, concerne 192000 élèves pour l'éducation primaire (+35000 qui fréquentent les écoles privées et publiques des pays hôtes); 53000 dans l'éducation secondaire (+ 30000 qui fréquentent les écoles privées et publiques des pays hôtes). Et l'insuffisance persistance du budget du programme amène le commissaire général de I'UNRWA, M Michelmore, à décider des coupes de $20 \%$ dans I'action éducative ${ }^{29}$.

\section{DE FORTES TENSIONS EN 1974}

En 1974, malgré l'opposition des États-Unis et d'Israël, I'Organisation de libération de la Palestine (OLP) est admise comme observateur avec droit à la parole à la conférence générale de I'Unesco. La conférence générale adopte en outre une résolution condamnant Israël pour ses fouilles dans la partie de Jérusalem occupée par cet État, fouilles jugées responsables d'avoir altéré la "physionomie historique» de la ville. En effet, Israël est partie à la convention pour la protection

27. Archives de I'Unesco, dossier 37:362.92 (5-011) UNRWA «67-», part VI : lettre de Samuel de Palma, département d'état américain, à Maheu, 3 fev 1971.

28. Archives de l'Unesco, dossier 37:362.92 (5-011) UNRWA «67-», part VI : lettre de $\mathrm{M}^{\prime}$ Bow, sous DG pour l'éduc, au secr gen de la fondation Agnelli (FIAT), 24 mai 71.

29. Archives de I'Unesco, dossier 37:362.92 (5-011) UNRWA «67--- », part IV: Appel de maheu, 1 janvier 1971. 
des biens culturels en cas de conflit armé, et cette convention interdit toute fouille et tous travaux dans des territoires occupés. La conférence générale adopte également une résolution condamnant Israël pour sa politique éducative et culturelle dans les territoires arabes occupés. Sur I'initiative de la plupart des pays musulmans et des pays communistes, la conférence générale décide de suspendre toute aide à Israël et refuse de classer Israël dans une région déterminée du monde (les Etats membres de I'Unesco sont classés par grandes régions du monde). Israël se retrouve ainsi « mise hors région $»^{30}$.

Israël réagit avec indignation à ce qui lui apparaît comme une provocation, et déclenche immédiatement une violente campagne de presse visant à discréditer l'Unesco. De nombreux intellectuels occidentaux protestent contre l'exclusion d'Israël $^{31}$. En décembre 1974, le Congrès américain exprime son indignation au cours de plusieurs séances $^{32}$. Les Etats-Unis, fermes soutiens d'Israël, protestent vivement contre cette décision. Cet épisode a des répercussions sur le programme Unesco-UNRWA : I'attitude d'Israël à l'égard de ce programme se tend. De plus, dans les années qui suivent cette crise, les Etats-Unis s'efforcent de réduire la part du budget attribué à I'UNRWA, arguant qu'il y aurait des gaspillages. Or, en 1978 les réfugiés palestiniens sont 1,7 millions. Les 595 écoles UNRWA-Unesco totalisent en 1977300000 élèves et plus de 10000 enseignants. II apparaît donc difficile de diminuer le budget de ce programme. Une mission envoyée par les Etats-Unis observe d'ailleurs "les mauvaises conditions des bâtiments scolaires et la surcharge qu'ils subissent » et conclut "que toute réduction du budget de I'UNRWA risque de provoquer des problèmes humanitaires, administratifs ou autre et qu'enfin dans la quasi-totalité des régions concernées, le gaspillage était chose inexistante $»^{33}$.

Devant cette volonté des Etats-Unis de restreindre les crédits qui lui sont attribués, le programme Unesco-UNRWA a du mal à équilibrer son budget. En 1975 les problèmes se poursuivent: les écoles du secteur manquent d'enseignants, et de locaux, les écoles détruites en 1967 n'ayant pas été reconstruites. En Jordanie, les autorités d'occupation ont démoli des écoles, plusieurs professeurs

30. 18 C/ Resol. 3.427 (1974) : protection des biens culturels à Jérusalem ; rejet de I'amendement israélien à la resol. 18 C/46.1 (1974).

31. Encyclopedia universalis, 1990, article "Unesco » par V.-Y. Ghebali.

32. Rapports du Congrès américain, Vol. 120, 93e congrès, 2e session, 21 janv-20 déc. $1974: 11$ déc. 1974, House, "Foreign assistance act of 1974 », p. 39129-39178.

33. Archives de I'Unesco, dossier 37 : 362.92 (5-011) «67--»: Analyse du rapport de la commission des relations étrangères au sénat des Etats-Unis sur I'UNRWA, juin 1978. 
et élèves ont été arrêtés et certains déportés, et dans le secteur jordanien de Jérusalem, les institutions scolaires gouvernementales ont été annexées au système d'éducation israélien contre le vœu et les intérêts de la communauté arabe ${ }^{34}$. Ainsi, à la suite de la guerre du Kippour (1973) et de l'admission de l'OLP comme observateur permanent à l'Unesco ainsi que de la « mise hors région » d'Israël par I'Unesco (1974), le programme éducatif UNRWA/Unesco est remis une nouvelle fois en question, souffrant de problèmes financiers et de démolitions, arrestations et annexions arbitraires de la part des autorités d'occupation israéliennes.

\section{LE DÉPLACEMENT DU SIÈGE DU PROGRAMME UNESCO- UNRWA À VIENNE}

En août 1978, en raison de la situation au Liban, le commissaire général de I'UNRWA décide de déplacer son quartier général qui était localisé à Beyrouth. II a la possibilité de l'établir à Damas, Amman, ou Vienne en Autriche. L'assistant directeur général de I'Unesco pour l'éducation, Sema Tanguiane, observe dans une lettre au DG qu' « un tel transfert (...) risque d'avoir des effets négatifs tant sur le plan pratique que sur le plan psychologique $»^{35}$. En 1979 le commissaire général de I'UNRWA est en négociation avec le gouvernement autrichien en vue de de transfert du siège de l'UNRWA à Vienne, qui sera effectué en dépit d'une résolution de l'assemblée générale de I'ONU lui demandant de regrouper le plus rapidement possible l'ensemble du siège dans la zone d'opérations, c'est-à-dire au Moyen-Orient ${ }^{36}$. Le DG de I'Unesco avait quant à lui refusé le transfert du département de I'éducation UNRWA-Unesco à Vienne, il est resté à Amman et souffre d'un manque de supervision en raison de la présence du commissaire général à Vienne. Ce changement de localisation du programme Unesco-UNRWA, ainsi qu'une réorganisation administrative interne, affaiblissent le fonctionnement de ce qui demeure, en 1979, le programme le plus important de I'Unesco dans le domaine de l'éducation scolaire.

Le DG de l'Unesco insiste pour que le personnel de I'Unesco affecté à I'UNRWA reste sur place. Le personnel de I'Unesco s'occupant de ce programme est envoyé à Amman. En novembre 1980, Sema Tanguiane,

34. Archives de I'Unesco, dossier 37:362.92 (5-011) UNRWA «67-», part VIII : note du 17 juill 75.

35. Archives de l'Unesco, dossier 37 : 362.92 (5-011) «67- - : Lettre de l'assistant directeur général pour l'éducation, S Tanguiane, au DG, 29 mai 1978. 36. Archives de I'Unesco, dossier $37: 362.92$ (5-011) «67-», part XIII : Mémo du sous DG pour l'éduc au DG, 5 fev 1979. 
assistant du DG de I'Unesco pour l'éducation, écrit au DG : « dans un câble récent (...) le représentant de I'UNRWA au Liban a signalé que le gouvernement libanais avait déclaré qu'il n'accepterait pas que I'UNRWA s'installe ailleurs qu'à Beyrouth, au cas où l'agence retournerait au Moyen Orient, et qu'il s'opposerait à l'installation éventuelle du siège de I'UNRWA à Amman $»^{37}$. La question de la localisation du siège du programme cause donc de fortes dissensions, qui sont dommageables au déroulement du programme. De plus, la situation financière du programme est difficile : dans cette même lettre, Sema Tanguiane observe que "le commissaire général de I'UNRWA est (...) très pessimiste quant à la situation financière de l'agence », et qu'il est « convaincu que des coupures dans le programme d'éducation seront nécessaires dans le courant de l'année prochaine. Sur cette lettre, à côté de ce passage, on trouve une note manuscrite du DG, qui a écrit «il faut les éviter à tout prix ! ${ }^{38}$, ce qui montre combien I'Unesco soutient ce programme éducatif.

Les coupes budgétaires, ainsi que la scission du siège de I'UNRWA entreVienne et Amman, ont des conséquences négatives pour l'efficacité du programme, comme l'observe Olof Rydbeck, commissaire général de l'UNRWA ${ }^{39}$.

\section{DES RÉUSSITES MAIS AUSSI TENSIONS ET DES PROBLÈMES FINANCIERS À LA FIN DES ANNÉES 1970}

A la suite de l'invasion israélienne du Sud-Liban qui a commencé vers la mi-mars 1978, I'enseignement est interrompu dans les établissements UNRWA/Unesco à I'exception de la Syrie. Comme le relate Sema Tanguiane au DG de I'Unesco, cette interruption a été provoquée au Liban par l'invasion et le déplacement des habitants du sud vers le nord et ailleurs par des manifestations d'étudiants et d'élèves. L'armée israélienne a pénétré de force dans les deux écoles normales UNRWA/Unesco de Ramallah; elle a emmené de force un professeur et quatre élèves et en a blessé deux ${ }^{40}$. Au Liban, les cours sont constamment interrompus chaque fois que des combats surviennent ou que les autorités militaires israéliennes entreprennent des raids de

37. Archives de I'Unesco, dossier 37 :362.92 (5-011) «67-», part XIV : Lettre de Tanguiane au DG, 10 nov 1980.

38. Archives de I'Unesco, dossier 37 :362.92 (5-011) « 67-», part XIV : Lettre de Tanguiane au DG, 10 nov 1980.

39. Archives de I'Unesco, dossier $37: 362.92$ (5-011) «67-», part XIV : Lettre de Olof Rydbeck à M'Bow, 19 juin 1980.

40. Archives de I'Unesco, dossier 37:362.92 (5-011) A 113/68 DG «69» part V : lettre de ADG/ED au DG, 5 mai 7 ? (non datée). 
représailles. L'occupation du Sud-Liban en mars/avril 1978 par Israël a entraîné l'arrêt complet de l'enseignement dans cette région ${ }^{41}$.

Par ailleurs I'UNRWA décide de suspendre à partir de juillet 1878 I'enseignement du premier cycle secondaire en raison du déficit budgétaire. Pour pallier ce déficit budgétaire, en décembre 1978 les Etats-Unis promettent 52 millions de dollars à I'UNRWA pour 1979. Et en décembre 1979, 41 gouvernements ont promis de verser à l'UNRWA des fonds, s'élevant à 91 millions dollars pour $1980^{42}$.

Le bilan à la fin des années 1970 apparaît malgré les difficultés assez positif. En 1979, ce programme, où la responsabilité technique de I'Unesco est devenue de plus en plus grande, concerne 320000 élèves inscrits dans 611 écoles avec 9200 enseignants. Aux améliorations quantitatives en effectifs se sont ajoutés des améliorations qualitatives au fil des années. les spécialistes des différentes disciplines mis par I'Unesco à la disposition de I'UNRWA donnent des conseils dans le domaine de la méthodologie de l'enseignement et offrent aux enseignants des cours de formation. De 1952 à 1978, les 7 centres d'enseignement technique et professionnel ont formé plus de 22000 techniciens. De 1956 à 1978, 4 écoles normales ont été créées, et 9000 enseignants en sont sortis diplômés.

Dans les années 1970, I'UNRWA et I'Unesco ont créé deux centres de développement de l'éducation - l'un à Amman et l'autre à Gaza - afin d'améliorer les niveaux d'enseignement dans ces régions. "On peut dire que le programme UNRWA/Unesco est maintenant comparable à n'importe quel système national d'éducation », conclut le DG de I'Unesco dans son rapport devant le conseil exécutif. Mais I'UNRWA est perpétuellement en proie à des difficultés financières ${ }^{43}$.

\section{DE GRAVES PROBLÈMES FINANCIERS AU DÉBUT DES ANNÉES 1980}

En 1980, I'UNRWA se rend compte que la situation financière prévue pour 1981 est " extrêmement préoccupante». "Tous les

41. Archives de I'Unesco, dossier 37:362.92 (5-011) UNRWA/EXBD part II : rapport oral du DG à la 107e session du cons ex, 9 avril 1979.

42. Archives de I'Unesco, dossier 37:362.92 (5-011) A 113/68 DG «69» part $\mathrm{V}$ : lettre de ADG/ED au DG, 5 mai 7 ? (non datée) ;press release de US Mission to the UN, 13 dec 1978 ; UNRWA news, dec 1979.

43. Archives de I'Unesco, dossier 37:362.92 (5-011) UNRWA/EXBD part II : rapport oral du DG à la 107e session du cons ex, 9 avril 1979 ; et 37:362.92 (5-011) A 113/68 DG « $69 »$ part V. 
services de I'UNRWA risquent de s'effondrer en mars 1981 ». Le programme d'éducation en Jordanie et en Syrie est en danger à cause des problèmes financiers. "Le seul espoir pour que les écoles continuent de fonctionner jusqu'à la fin de 1980 réside dans les informations encore non confirmées et suivant lesquelles les Etats-Unis verseraient quelques millions de dollars supplémentaires », observe un agent en juillet $1980^{44}$. II s'avère nécessaire pour combler le déficit de fermer des écoles. II est envisagé de lancer un appel ciblé à quelques Etats membres pour donner des contributions supplémentaires ${ }^{45}$.

En février 1981, le DG de I'Unesco, Amadou Mahtar M'Bow, déplore que $M$. Rydbeck, responsable de I'UNRWA, ait décidé "sans consultation préalable de l'Unesco », de fermer les 635 écoles élémentaires et préparatoires de l'ensemble des zones d'opération de I'UNRWA. II rappelle aussi qu'il était contre scission et transfert à Vienne et demande à ce que tout soit réunifié sur place ${ }^{46}$. Ainsi on observe de fortes dissensions entre I'Unesco et I'UNRWA.

A la fin de l'année 1981, le problème financier est tout aussi grave. L'UNRWA est au bord de l'effondrement, risquant un déficit de 80 millions pour $1982^{47}$.

\section{DES TROUBLES ACCRUS AU DÉBUT DES ANNÉES 1980}

Les mauvaises conditions de travail dans les écoles UnescoUNRWA, liées notamment aux problèmes budgétaires, entraînent des troubles dans les années 1980. De décembre 1980 à février 1981, les 99 écoles et trois centres de formation Unesco-UNRWA dans la bande de Gaza sont fermés pour cause de grève des enseignants ${ }^{48}$.

De plus, en juillet 1980, le gouvernement militaire israélien prend des mesures visant à renforcer son contrôle sur tous les établissements d'enseignement de la Cisjordanie occupée en promulguant une

44. Archives de I'Unesco, dossier 37:362.92 (5-011) A 113/68 DG « 69 » part V : traduction du télégramme de Feridun, UNRWA, Vienne, du 22 juill 1980.

45. Archives de I'Unesco, dossier 37:362.92 (5-011) UNRWA « 67 -», part VIII : note d'information à l'intention du DG, 21 janv 1981.

46. Archives de I'Unesco, dossier 37:362.92 (5-011) A 113/68 DG « 69 » part VI : lettre de M'Bow à Olof Rydbeck, commissaire général UNRWA, 19 fev 1981.

47. Archives de I'Unesco, dossier 37:362.92 (5-011) A 113/68 DG « 69 » part VII : UNRWA news, oct 1981.

48. Archives de I'Unesco, dossier 37 :362.92 (5-011) «67-», part XIV : UNRWA press release $\mathrm{n}^{\circ} 1 / 81,12$ fev 1981 . 
série d'ordonnances militaires, notamment l'ordonnance militaire $n^{\circ} 854$. Le conseil exécutif de l'Unesco observe que ces ordonnances compromettent l'existence des universités, enfreignent le principe de l'enseignement obligatoire, réduisent le nombre des enseignants potentiels, soumettent le processus éducatif à des «manipulations politiques ", rendent précaire le statut des élèves et des enseignants, et que de plus elles sont contraires à la convention de Genève, qu'elles contribuent à l'exode des compétences, et qu'elles instaurent une tension permanente au sein des universités ${ }^{49}$.

En novembre 1981, I'observateur permanent de I'OLP à I'Unesco écrit au DG de I'Unesco, se plaignant d'une amplification des persécutions dans les territoires arabes occupés, et déplorant la fermeture de I'université de Beir-Zeit. Le DG de I'Unesco demande alors au ministre des affaires étrangères d'Israël que l'université de Beir Zeit soit rouverte ${ }^{50}$.

Le 1 er décembre 1981, le ministre de l'éducation de la République arabe syrienne écrit au DG de l'Unesco pour lui faire part de sa préoccupation au sujet de la situation éducative et culturelle dans le Golan occupé. II déclare que les autorités israéliennes détruisent l'identité culturelle arabe et réduisent l'éducation dans les territoires arabes occupés à un niveau très bas, orientant la population vers une formation professionnelle minimale "de façon à l'exploiter ... comme main d'œuvre bon marché », dans l'économie israélienne. Il observe que les autorités israéliennes ont changé les programmes, les ont remplacés par des programmes "axés sur l'enseignement de I'hébreu et I'histoire du peuple juif, négligeant l'enseignement de I'arabe et l'histoire des Arabes, et introduisant de graves déformations destinées à effacer le caractère arabe des programmes et à détruire I'identité culturelle des étudiants arabes ». Le DG de I'Unesco sert, sur cette question, d'intermédiaire entre la déléguée permanente d'Israël à I'Unesco et les représentants des pays arabes ${ }^{51}$.

En février 1982, le DG de I'Unesco écrit au ministre des affaires étrangères d'Israël pour lui demander de rouvrir université de Beir-Zeit. Sur ces entrefaites, I'invasion israélienne du Liban entraîne des troubles accrus pour les écoles et centres de formation Unesco-UNRWA situés dans ce pays.

49. Archives de I'Unesco, dossier 37:362.92 (5-011) UNRWA/EXBD part II : 114EX/13, annexe III.

50. Archives de I'Unesco, dossier 37:362.92 (5-011) (074) part IX : lettre de M'Bow à madame Yael Vered, 1981.

51. Archives de I'Unesco, dossier 37:362.92 (5-011) UNRWA/EXBD part II : 114 EX/13, Paris, 22 avril 1982. 
Plusieurs interruptions des cours ont lieu en mars 1982 ainsi que des arrestations et emprisonnement d'étudiant(e)s de Ramallah ${ }^{52}$. Le danger et la peur sont quotidiens. En avril 1982, le DG de l'Unesco informe le conseil exécutif sur la situation dans les écoles UNRWA-Unesco : de nombreux maîtres n'ont reçu qu'une formation initiale médiocre; on manque de matériel didactique ; les classes sont fréquemment surchargées et les locaux sont souvent peu adaptés ou en mauvais état ; la condition du personnel enseignant devient précaire financièrement car l'évolution des traitements de ce personnel ne suit pas en effet l'augmentation du coût de la vie ; de plus, les enseignants ne peuvent pas créer ses propres syndicats. II conclut que la qualité de l'enseignement dispensé souffre grandement d'une telle situation. De plus, c'est le système lui-même qui semble pervers, contribuant à ce que les élèves de ces écoles soient ensuite exploités économiquement par Israël : en effet le système de formation professionnelle mis en place vise à former rapidement « une main d'œuvre palestinienne semi-qualifiée immédiatement utilisable et recrutée, le plus souvent, par des entreprises situées en Israël ». En outre, "I'insuffisance des salaires du personnel enseignant l'oblige souvent à exercer une seconde activité professionnelle, ce qui nuit à la qualité de ses services et à son image sociale $»^{53}$.

\section{DESCONFLITSQUISECONCENTRENTSURLESINSTITUTIONS UNIVERSITAIRES DANS LES ANNÉES 1980}

En juillet 1982, le Dr Hanna Nasir, président en exil de l'université de Beir-Zeit, envoie un télégramme au DG pour l'informer que l'université de Beir-Zeit venait d'être fermée pour une durée de trois mois sur ordre des autorités militaires israéliennes, ceci pour la troisième fois pendant I'année universitaire en cours (1981-82). En ce qui concerne les circonstances de cette fermeture, il indique que certains étudiants et d'autres membres de la communauté de Cisjordanie ont effectivement protesté contre l'invasion israélienne du Liban, mais souligne que la protestation était de caractère pacifique et n'avait pas eu lieu sur le campus universitaire. Hanna Nasir demande au DG d'intervenir en vue d'obtenir la réouverture de l'université. En juillet 1982 le DG a alors écrit au ministre des affaires étrangères d'Israël à ce sujet, puis en octobre 1982 à nouveau ${ }^{54}$.

52. Archives de I'Unesco, dossier 37:362.92 (5-011) UNRWA/EXBD part II : 114EX/13 Add.2, 11 mai 1982.

53. Archives de l'Unesco, dossier 37:362.92 (5-011) UNRWA/EXBD part II : éléments pour l'information du conseil exécutif par le DG sur la mission dans les territoires arabes occupés et certaines de ses conclusions, 21 mars-6 avril 1982. 54. Archives de l'Unesco, dossier 37:362.92 (5-011) UNRWA/EXBD part II : 116EX/16, Paris, 16 mai 1983. 
Un document de l'Unesco du 30 août 1982 observe que 175000 réfugiés auraient été obligés de quitter le sud du Liban et la ville de Beyrouth ; qu'une ou deux écoles ont été rasées, mais que la plupart d'entre elles pourraient être réouvertes après évacuation. II note aussi que les professeurs sont dispersés et que certains ont été détenus par I'armée israélienne. Face à cette situation, I'UNRWA/Unesco a agi, créant à partir de mi-juillet cinq task forces à Tyr, Beyrouth, Tripoli, Saisa et la Békaa pour essayer de recenser les étudiants et les enseignants ${ }^{55}$. A cette époque, I'effectif total du programme Unesco-UNRWA dépasse 339000 élèves, 5000 étudiants et 9800 enseignants $^{56}$. Le DG de I'Unesco fait l'intermédiaire entre les représentants arabes et israéliens pour essayer de régler cette situation de crise.

En septembre 1982, Sema Tanguiane observe que les autorités d'occupation israéliennes imposent aux enseignants universitaires étrangers dans les territoires occupés comme condition à la délivrance $d^{\prime}$ un permis de travail la signature $d^{\prime}$ 'une déclaration par laquelle ils déclarent s'engager « pleinement à se refuser à tout acte offrant quelque assistance que ce soit à l'Organisation appelée OLP ou toute autre organisation terroriste considérée comme hostile à I'Etat d'Israël ». De ce fait, 25 membres de la faculté de Najah et trois membres de la faculté de Bethléem, ayant refusé de signer cette déclaration, n'ont $p$. obtenir de permis de travail et sont obligés de quitter le pays. Cinquante autres, dans les centres universitaires, sont menacés. L'Unesco juge qu'Israël n'a pas à demander un tel engagement, car l'OLP est une organisation de libération nationale reconnue par la communauté internationale ${ }^{57}$. Finalement, en novembre 1982, suite à la pression du secrétaire d'état des Etats-Unis, les autorités israéliennes annulent l'exigence de cet engagement écrit ${ }^{58}$.

En juin 1983, I'observateur permanent de l'OLP auprès de I'Unesco se plaint au DG de l'Unesco de la fermeture de l'université d'al Najah sur ordre d'Israël et de l'expulsion de 28 de ses enseignants dont le président de l'université et son adjoint, sous prétexte qu'ils ont refusé

55. Archives de I'Unesco, dossier 37 :362.92 (5-011) «67-», part XV : Doc du 30 août 1982, intitulé : " aide aux réfugiés et aux mouvements de libération $»$.

56. Archives de I'Unesco, dossier $37: 362.92$ (5-011) «67-», part XV : Rapport oral du DG à la 115e session du conseil exécutif.

57. Archives de I'Unesco, dossier 37:362.92 (5-011) (074) part X : lettre du ADG/ED au DG, 29 septembre 1982.

58. Archives de I'Unesco, dossier 37:362.92 (5-011) (074) part X : 22 nov 1982, lettre de Tanguiane au DG. 
de signer un document qui condamne l'OLP ${ }^{59}$. Le mois suivant, ce même observateur déplore que quatre étudiants de l'université islamique d'Hébron en Cisjordanie ont été tués par les Israéliens, et que le lendemain une étudiante ait tuée par une balle tirée par un soldat israélien, pour avoir participé à une manifestation protestant contre I'attentat d'Hébron; le jour suivant, quatre étudiants de l'université de Beir Zeit, manifestant au sujet de ces événements, sont blessés par balles tirées par les forces israéliennes ${ }^{60}$.

La situation devient de plus en plus tendue, I'hostilité entre les deux camps aboutissant à des morts. En janvier 1984, le centre de formation des instituteurs à Ramallah est fermé. En avril 1984, Mohieddine Saber, DG de I'ALECSO (I'organisation des Etats arabes pour l'éducation, la science et la culture, structure créée sur le modèle de l'Unesco pour les Etats arabes) écrit au DG de l'Unesco : il lui demande d'agir, indigné que "des étudiants meurent assassinés simplement parce qu'ils sont palestiniens et osent revendiquer l'exercice des droits de I'homme $»^{61}$. En août 1984, dans un télégramme, le DG de I'Unesco demande instamment au ministre des affaires étrangères $d^{\prime}$ Israël de rouvrir l'université al Najah'². En mars 1985, Omar Massalha, observateur permanent de I'OLP à I'Unesco, écrit au DG de I'Unesco, lui apprenant que les Israéliens ont décidé, pour la 10e fois, une nouvelle fermeture de l'université de Beir Zeit. De plus, «le 21 novembre dernier, I'armée israélienne a ouvert le feu aux étudiants de cette université qui manifestaient pacifiquement». II ajoute: "la semaine dernière les autorités de l'occupation ont entravé également le fonctionnement normal de l'université de Al Najah, en l'encerclant par une centaine de soldats et en installant des barrières de contrôle aux entrées de l'université, pour humilier ses enseignants et ses étudiants ». Il lui demande d'intervenir. En réponse, le DG de l'Unesco adresse un nouveau télégramme au ministre des affaires étrangères d'Israël pour lui demander que l'université de Beir Zeit soit rouverte ${ }^{63}$.

59. Archives de l'Unesco, dossier 37:362.92 (5-011) (074) part X : lettre de I'observateur permanent de I'OLP aupres de I'Unesco, au DG, 8 juin 1983. 60. Archives de I'Unesco, dossier 37:362.92 (5-011) (074) part X : lettre de I'observateur permanent de I'OLP auprès de I'Unesco, au DG, 29 juill 1983. 61. Archives de I'Unesco, dossier 37:362.92 (5-011) (074) part X : lettre de Mohieddine Saber, DG Alecso, au DG de I'Unesco, 5 avril 1984.

62. Archives de I'Unesco, dossier 37:362.92 (5-011) (074) part X: 9 août 1984 : télégramme du DG Unesco au ministre des affaires étrangères d'Israël. 63. Archives de I'Unesco, dossier 37:362.92 (5-011) (074) part X : lettre d'Omar Massalha, observateur permanent de I'OLP à I'Unesco, au DG, 7 mars 1985 ; lettre de M’Bow à Massalha, non datée. 
En avril 1985, Meir Shamir, ministre plénipotentiaire, délégué permanent d'Israël auprès de l'Unesco, écrit au DG de I'Unesco, et lui donne sa version des faits : " les autorités de Judée et Samarie ont eu connaissance de la préparation au nouveau campus de l'université de Beir-Zeit d'un certain anniversaire de l'organisation terroriste de Nayef Hawatme, qui a sur sa conscience des massacres de civils »; du matériel a été trouvé, notamment des livres expliquant comment faire un cocktail Molotov et des livres " célébrant la tuerie d'Israéliens ", " des affiches et photos glorifiant des terroristes » et des slogans « mort aux juifs $»^{64}$. Ainsi les Israéliens se sentent attaqués, et ont le sentiment d'être en droit de répliquer à ce qu'il perçoivent comme des provocations. Le conflit se focalise sur la question de la fermeture des universités où étudient les jeunes Palestiniens. En 1984, la conférence générale de I'Unesco adopte une résolution affirmant la volonté d'obtenir la réouverture de l'université de Bethléem et des autres institutions éducatives fermées. Ainsi, au début des années 1980, du fait des tensions accrues entre Israéliens et Palestiniens, le programme Unesco/UNRWA connaît de sérieuses difficultés concernant sa branche universitaire.

\section{CONCLUSION}

Malgré les difficultés immenses dues à la situation de tension et même par moments de guerre entre les deux communautés ennemies qui s'affrontent sur le territoire palestinien, le programme éducatif UNRWA/Unesco a, de sa création en 1949 jusqu'aux années 1980, réalisé beaucoup de choses, mettant sur pied un véritable système scolaire pour les jeunes Palestiniens. En 1972, ce système rassemble près de 250000 élèves dans 500 écoles, avec 7000 enseignants. En 1975, I'UNRWA dépense 45 millions de dollars pour l'exécution de ses programmes d'éducation menés conjointement avec I'Unesco.

Les moments de conflits violents (1956, 1967, 1974 et à partir de 1982 en particulier) ont perturbé gravement le fonctionnement de ce système et dans ces moments, I'Unesco a p. apparaître impuissante à régler les tensions, mais, malgré les nombreuses difficultés, notamment financières, ce programme a toujours continué à œuvrer à la scolarisation des jeunes Palestiniens.

L'établissement d'universités ouvertes aux étudiants palestiniens atteste du succès de ce programme. Toutefois, à la fin des années

64. Archives de I'Unesco, dossier 37:362.92 (5-011) (074) part X : lettre de Meir Shamir, ministre plénipotentiaire, délégué permanent d'Israël auprès de I'Unesco, au DG Unesco, 2 avril 1985. 
1970 et au début des années 1980, le système universitaire souffre de fermetures et d'arrestations arbitraires de la part des Israéliens. Mais en dépit des conflits parfois violents, I'université de Bethléem a continué à fonctionner: malgré douze fermetures ordonnées par l'armée israélienne, dont la plus longue, de trois ans, entre octobre 1987 et octobre 1990, les cours ont toujours été assurés. De nos jours, cette université accueille près de 2600 étudiants inscrits.

Aujourd'hui I'UNRWA, qui existe toujours, est de loin la plus grande agence des Nations Unies, avec un personnel de plus de 25000 employés, essentiellement des réfugiés palestiniens recrutés localement et œuvrant comme enseignants, médecins ou travailleurs sociaux. 